\title{
Impact of enhanced recovery after surgery protocol compliance on patients' outcome in benign hysterectomy and establishment of a predictive nomogram model
}

Yiwei Shen ${ }^{1}$, Feng Lv' ${ }^{1}$, Su Min ${ }^{1 *} \mathbb{0}$, Gangming Wu${ }^{1}$, Juying Jin' ${ }^{1}$, Yao Gong ${ }^{2}$, Jian Yu², Peipei Qin ${ }^{1}$ and Ying Zhang ${ }^{1}$

\begin{abstract}
Background: Enhanced recovery after surgery (ERAS) pathways have been shown to improve clinical outcomes after surgery. Considering the importance of patient experience for patients with benign surgery, this study evaluated whether improved compliance with ERAS protocol modified for gynecological surgery which recommended by the ERAS Society is associated with better clinical outcomes and patient experience, and to determine the influence of compliance with each ERAS element on patients' outcome after benign hysterectomy.

Methods: A prospective observational study was performed on the women who underwent hysterectomy between 2019 and 2020. A total of 475 women greater 18 years old were classified into three groups according to their per cent compliance with ERAS protocols: Group I: <60\% (148 cases); Group II: $\geq 60$ and < 80\% (160 cases); Group III: $\geq 80 \%$ (167 cases). Primary outcome was the 30-day postoperative complications. Second outcomes included QoR-15 questionnaire scores, patient satisfaction on a scale from 1 to 7 , and length of stay after operation. After multivariable binary logistic regression analyse, a nomogram model was established to predict the incidence of having a postoperative complication with individual ERAS element compliance.

Results: The study enrolled 585 patients, and 475 completed the follow-up assessment. Patients with compliance over $80 \%$ had a significant reduction in postoperative complications ( $20.4 \%$ vs $41.2 \%$ vs $38.1 \%, P<0.001$ ) and length of stay after surgery ( 4 vs 5 vs $4, P<0.001$ ). Increased compliance was also associated with higher patient satisfaction and QoR-15 scores $(P<0.001)$,. Among the five dimensions of the QoR-15, physical comfort $(P<0.05)$, physical independence $(P<0.05)$, and pain dimension $(P<0.05)$ were better in the higher compliance groups. Minimally invasive surgery (MIS) $(P<0.001)$, postoperative nausea and vomiting (PONV) prophylaxis $(P<0.001)$, early mobilization $(P=0.031)$, early oral nutrition $(P=0.012)$, and early removal of urinary drainage $(P<0.001)$ were significantly associated with less complications. Having a postoperative complication was better predicted by the proposed nomogram model with high AUC value (0.906) and sensitivity (0.948) in the cohort.
\end{abstract}

\footnotetext{
*Correspondence: ms89011068@163.com

1 Department of Anesthesiology, The First Affiliated Hospital

of Chongqing Medical University, No.1 Youyi Road, Yuzhong District,

Chongqing 400016, People's Republic of China

Full list of author information is available at the end of the article
}

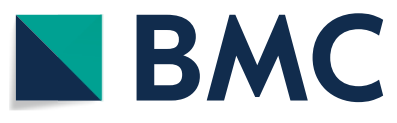

(c) The Author(s) 2021. Open Access This article is licensed under a Creative Commons Attribution 4.0 International License, which permits use, sharing, adaptation, distribution and reproduction in any medium or format, as long as you give appropriate credit to the original author(s) and the source, provide a link to the Creative Commons licence, and indicate if changes were made. The images or other third party material in this article are included in the article's Creative Commons licence, unless indicated otherwise in a credit line to the material. If material is not included in the article's Creative Commons licence and your intended use is not permitted by statutory regulation or exceeds the permitted use, you will need to obtain permission directly from the copyright holder. To view a copy of this licence, visit http://creativecommons.org/licenses/by/4.0/. The Creative Commons Public Domain Dedication waiver (http://creativecommons.org/publicdomain/zero/1.0/) applies to the data made available in this article, unless otherwise stated in a credit line to the data. 
Conclusions: Improved compliance with the ERAS protocol was associated with improved recovery and better patient experience undergoing hysterectomy. MIS, PONV prophylaxis, early mobilization, early oral intake, and early removal of urinary drainage were of concern in reducing postoperative complications.

Trial registration: Chinese Clinical Trial Registry, ChiCTR1800019178. Registered on 30/10/2018.

Keywords: Enhanced recovery after surgery (ERAS), Hysterectomy, Compliance, Postoperative complications, Patient satisfaction, Patient experience

\section{Background}

Enhanced recovery after surgery (ERAS) pathway is a multimodal perioperative care approach, designed to reduce perioperative stress and shorten recovery time [1]. The success of the ERAS pathway is attributed to the synergy between its elements [2, 3]. Although ERAS protocols comprise several different perioperative interventions, not every participant is able to complete the protocols. Implementation and adherence to the protocol is crucial to achieve, [4-6] with the challenges including lack of knowledge, resistance to change, and shortage of staff [7]. A growing number of researches have confirmed that the compliance with ERAS protocols is associated with postoperative rehabilitation in patients undergoing colorectal, orthopedic and other surgeries $[8,9]$.

Hysterectomy on benign indication is the most common major gynecological surgery performed worldwide [10]. The aim of surgery for benign diseases is primarily to improve the quality of life related to health, and high-quality recovery and good patient experience after surgery are therefore very important for both patients and society. Patient-reported outcomes are the patientcentered way to track and study all stages of surgical recovery across multiple domains and longitudinal time span [11].

Recently, enhanced recovery protocols have been introduced in general settings for gynecological surgeries [1215]. However, to date, no clinical studies have focused on ERAS protocol compliance for benign hysterectomy, particularly patient self-assessment and satisfaction. This study aims to evaluate whether improved compliance with ERAS protocol modified for gynecological surgery is associated with better clinical outcomes and patient experience, and to determine the impact of compliance with each ERAS element on patient outcomes after hysterectomy. In addition, we developed a model to predict the incidence of having a postoperative complication with individual ERAS element compliance.

\section{Methods}

This study was approved and conducted by the local Ethics Committee of the First Affiliated Hospital of Chongqing Medical University (No.2019-020), and registered with ClinicalTrials.gov (ChiCTR1800019178,
30/10/2018). Patients were informed of the ERAS protocols and signed the informed consent form before study entry, and the study was conducted in accordance with the Declaration of Helsinki. A prospective observational study on patients undergoing benign hysterectomy was performed at the Department of Gynecology in the First Affiliated Hospital of Chongqing Medical University in China during the period February 1st 2019 to December 31st 2020, with the ERAS protocols underwent.

\section{Study design}

Inclusion criteria for the subjects were age more than 18 years old with elective open or laparoscopic hysterectomy for benign conditions. Exclusion criteria were genital prolapse as indication for the hysterectomy, previous bilateral oophorectomy or the present operation would leave the woman without ovaries, physically or mentally disabled, severe psychiatric disease, or informed consent could not be obtained. All hysterectomies were total, but the type of surgical procedure was up to the operating surgeon. The criteria for laparoscopy or laparotomy were based on the Clinical guidelines for treatment $[16,17]$. To avoid the influence of radiotherapy or chemotherapy on patients experience and outcomes, patients with malignant and borderline tumors were excluded. Six surgeons, twelve nurses, five anaesthetists, one dieticians, and three physiotherapists formed the ERAS multi-disciplinary team (MDT), which effectively implemented the ERAS protocols. The protocols are based on the practice guidelines for gynecologic surgery by the ERAS society [16-18] and consist of 22 items involving preoperative, intraoperative and postoperative interventions (Additional file 1). All treatments were performed by ERAS MDT. To ensure that the protocols were running smoothly, everyone on the team communicated with each other and reported their work at weekly meetings. The study protocol conforms to GCP (Good Clinical Practice) standard procedures, and all investigators were trained and certified.

The Department of Gynecology of the First Affiliated Hospital of Chongqing Medical University serves a large geographic area in western and southwestern China with a referral base of 2 million people. The first gynecological oncology guidelines recommended by the ERAS Society were published in 2016 [18, 19] and revised in 2019 
[20], which was formally carried out in our hospital from October in 2016. Every patients would receive a "rehabilitation log" which containing perioperative ERAS guidelines and survey on each items. Periodic text message reminders were sent to remind participants about completion of the rehabilitation logs every day, such as early mobilization and early oral intake. For example, on the first postoperative day, in addition to the pre-operative education by nurses, mobile phone messages would remind patients that they should have an out-of-bed activity for $2 \mathbf{h}$.

The implementation of each item for each patient and outcome were collected prospectively. For categorical elements compliance was marked as yes/no. For intravenous fluids on the first postoperative day, ERAS compliance with balanced fluids was set to less than $2000 \mathrm{ml}$. The compliance rate for each patient was calculated as the number of interventions fulfilled/22 (total number of ERAS items). Mean total compliance was calculated as the average of all perioperative ERAS interventions. Patients were categorized into three groups by their compliance rates with the ERAS pathway. The same cutoff values as previous studies were used, [21, 22] where compliance was classified as 'poor', 'partial', or 'full' when $<50 \%, \geq 50 \%$, or $\geq 80 \%$. However, in the pilot trial before the formal start of this study, only a very small number of patients had a compliance rate $<50 \%$, so the poor group was classified as $<60 \%$. Each patient was followed by the ERAS team members during the hospital stay.

Characteristics of the study population, age, body mass index (BMI), education level, smoking status, nutritional risk screening (NRS) score, NYHA status, ASA status, history of surgery, preoperative hemoglobin, presence of chronic pain, history of postoperative nausea and vomiting (PONV), diagnosis and type of surgical procedure were recorded. Preoperative comorbidities were also recorded, such as hypertension, diabetes, chronic obstructive pulmonary disease (COPD), ischemic heart disease and asthma. After entering the operating room, the plasma electrolyte level was monitored by invasive arterial pressure puncture. Preoperative hypokalemia was defined as less than $3.5 \mathrm{mmol} / \mathrm{L}$. Intraoperative data were prospectively collected, including duration of surgery (min), intraoperative net fluid input and blood loss.

All of the patients were asked to complete 15-item quality of recovery (QoR-15) questionnaire, a widely used self-rated questionnaire for early postoperative quality of recovery [23, 24] (Additional file 2), under the guidance of the investigator on the day before operation, then to repeat it $24 \mathrm{~h} / 48 \mathrm{~h} / 72 \mathrm{~h}$ postoperatively [25]. QoR-15 is a patient-centered comprehensive questionnaire (15-items), which includes five aspects: physical comfort (5-items), emotional status (4-items), physical independence (2-items), psychological support (2-items) and pain (2-items) [26]. Total scores of the QoR-15 ranges from 0 to 150 , higher score indicates better recovery. At the same time, their resting pain was also measured with an 11-pointed visual analogue scale (VAS) ranging from 0 to 10 , with 0 indicating no pain and 10 indicating the worst pain imaginable. VAS $\geq 4$ was considered to identify patients with postoperative pain of moderate-to-severe intensity. The Likert scale (strongly dissatisfied $=1$, moderately dissatisfied $=2$, slightly dissatisfied $=3$, neutral $=4$, slightly satisfied $=5$, moderately satisfied $=6$, extremely satisfied $=7$ ) was used to evaluate patient satisfaction on the day of discharge and 30 days after discharge [27]. On this instrument, a higher score indicates a higher level of satisfaction.

Patients were discharged when they met the strict criteria: mobilization with normal diet, oral analgesics for pain relief, normal urination and no intestinal obstruction signs. Patients were contacted by a dedicated nurse on the telephone at 30 days after discharge. The readmission, postoperative chronic complications and patient satisfaction were also collected.

\section{Study outcomes}

Primary outcome of the study was the incidence of postoperative complications, including PONV, moderate-tosevere postoperative pain, deep vein thrombosis (DVT), surgical site infection, and pulmonary infection. Postoperative complications were monitored until 30 days after surgery, which were defined by the guidelines for European perioperative clinical outcome definitions [28], as shown in Additional file 3.

Secondary outcomes included patient satisfaction, QoR-15 scores (including five dimensions), LOS after surgery, mortality, postoperative hospitalization costs, readmission rate within 30 days post-discharge. LOS after surgery was defined as the number of days patients stayed in the hospital after operation. Postoperative hospital costs were presented as RMB converted to Euro (November 2020), which were obtained from hospital databases.

\section{Statistical analysis}

The characteristics of study participants in the three groups were described with a descriptive analysis, while the normal distribution of the data was checked with Shapiro-Wilk test. Outliers were investigated and eliminated in the event of a demonstrably incorrect measurement or input error. Categorical variables were described as numbers with percentages, and continuous variables were described as the means with standard deviations (SD) or median and interquartile range (IQR), depending on the distribution, which was checked through visual inspection of the histogram. The Chi square test, 
repeated measures analysis of variance, Mann-Whitney $\mathrm{U}$ test and Kruskal-Wallis test were used to compare the outcomes between groups where appropriate. Fisher's exact test was used for categorical variables when the number of events was less than five.

Independent of the previous analysis, patients were divided into two groups based on the presence or absence of postoperative complications. All the factors including the clinicopathological factors and each component of ERAS were put into the univariable logistic regression to analyze their correlation with the probability of having any postoperative complication. Then the factors with $P$ value less than 0.05 were included in the multivariable binary logistic regression and hazard ratios of each factors were calculated. Through the multivariable binary logistic regression analysis, the factors with $P$ value less than 0.05 were selected to develop a nomogram prediction model by $\mathrm{R}$ software. The discrimination performance of the nomogram model was quantified with receiver operation characteristic (ROC) curve, with the value of the area under the curve (AUC) of the ROC curve between 0.5 and 1.0, and the closer the AUC value is to 1 , the better discrimination performance the model has. Calibration curves were plotted to assess the calibration of the nomogram model.

A sample size calculation was based on the primary endpoint, the incidence of postoperative complications, as the previous study had reported that the incidence of postoperative complications undergoing gynecological operation was approximately $25 \%$ [2]. The requirement of minimum sample size for each group in this study was 118 participants to reach the statistical significance at two-sided $95 \%$ confidence interval and setting the power to $90 \%$. To account for predicted dropout rate of $20 \%$, we decided to recruit 542 patients. The sample size calculation was performed PASS 15.0 analysis program.

Statistical analyses were completed in SPSS for Windows version 23.0 (SPSS, Inc., Chicago, IL, USA) and GraphPad Prism for Windows version 8.0 (GraphPad Software, Inc., San Francisco, CA, USA). The nomogram plot, ROC curve and calibration curve were plotted using $\mathrm{R}$ software (version 3.6.2) with RMS, ROCR package( $\mathrm{R}$ Core Team, Vienna, Austria). A 2 -sided $P$ value $<0.05$ was regarded as statistically significant.

\section{Results}

\section{Patient characteristics}

A total of 585 women were screened for eligibility from February 2019 to December 2020, of whom, 542 were enrolled (showed in Fig. 1). Twenty one women dropped out during the study, leaving 521 women for short-term outcome analyses. Moreover, 46 women were lost during the post-discharge follow-up, allowing for long-term outcomes analyses of 475 women, ranging from 37 to 70 years old, with the median age 49 years old. The overall compliance rate of ERAS protocol modified for gynecological surgery in this study was $80.6 \%$. According to the compliance with the

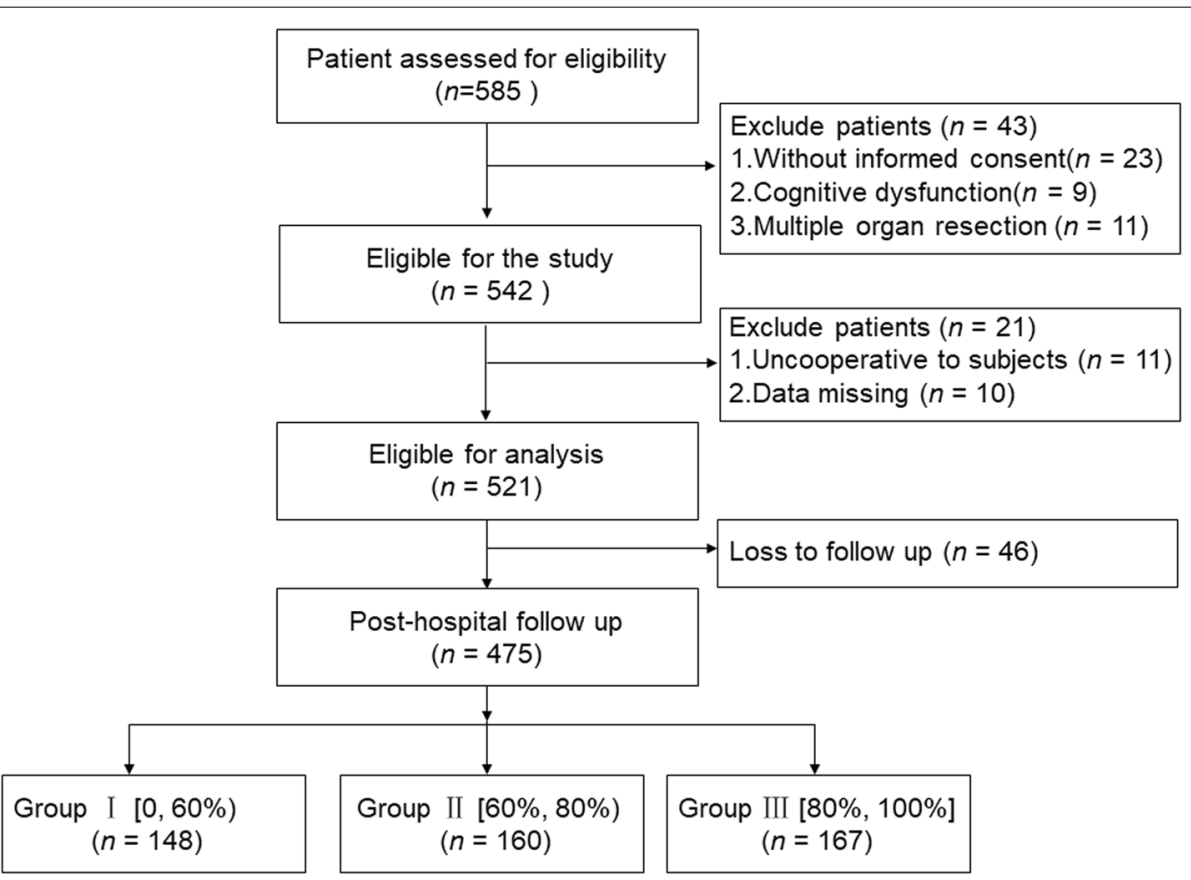

Fig. 1 Flowchart of the study participant selection 
ERAS protocols, women were categorized into three groups: Group I included women with compliance less than $60 \%$; Group II, those with 60 to $80 \%$ compliance; and Group III, women with more than $80 \%$ compliance. The number of patients in each group was 148 , 160 , and 167.
No significant differences in patient demographics among the three groups was found, including age, BMI, smoking status, NRS score, ASA status, NYHA status, comorbidity, history of previous lower abdominal surgery and PONV. Baseline assessments and surgical characteristics of the study population are shown in Table 1.

Table 1 Baseline Assessments and Surgical Characteristics of the Study Population

\begin{tabular}{|c|c|c|c|c|}
\hline Compliance & $\begin{array}{l}\mathrm{I}[0,60 \%) \\
(N=148)\end{array}$ & $\begin{array}{l}I I[60,80 \%) \\
(N=160)\end{array}$ & $\begin{array}{l}\mathrm{III}[80,100 \%] \\
(N=167)\end{array}$ & $P$ value \\
\hline Age $\left(\right.$ years $\left.{ }^{\mathrm{a}}\right)$, median $(\mathrm{QQR})$ & $47.0(44-52)$ & $50.0(43-54)$ & $49.0(47-52)$ & $0.323 \triangle$ \\
\hline BMI $\left(\mathrm{kg} / \mathrm{m}^{2 \mathrm{a}}\right)$, median (IQR) & $23.7(22.5-26.6)$ & $25.1(22.0-26.1)$ & $23.4(22.2-25.1)$ & $0.303 \triangle$ \\
\hline Education level above high school, $n(\%)$ & $101(68)$ & $103(64)$ & $98(59)$ & 0.558 \\
\hline \multicolumn{5}{|l|}{ Smoking status, $n(\%)$} \\
\hline Never smoked & 140(95) & $155(97)$ & 158(95) & 0.259 \\
\hline Current smoked & $2(1)$ & $1(0)$ & $3(2)$ & $0.451^{*}$ \\
\hline Former smoked & $6(4)$ & $4(3)$ & $6(4)$ & $0.507^{*}$ \\
\hline NRS score, $n(\%)$ & & & & 0.398 \\
\hline$N R S<3$ & $98(66)$ & $94(59)$ & $105(63)$ & \\
\hline$N R S \geq 3$ & $50(34)$ & $66(41)$ & $62(37)$ & \\
\hline \multicolumn{5}{|l|}{ Comorbidities, $n(\%)$} \\
\hline Hypertension & 28(19) & $26(16)$ & $25(15)$ & 0.635 \\
\hline Diabetes & $10(7)$ & $6(4)$ & $12(7)$ & 0.363 \\
\hline COPD & $4(3)$ & $8(5)$ & $5(3)$ & $0.523^{*}$ \\
\hline Ischemic heart disease & $3(2)$ & $3(2)$ & $5(3)$ & $0.348^{*}$ \\
\hline Asthma & $5(3)$ & $2(1)$ & $2(1)$ & $0.120^{*}$ \\
\hline NYHA status, $n(\%)$ & & & & 0.558 \\
\hline 1 & $125(84)$ & $144(90)$ & 149(89) & \\
\hline$\|$ & $23(16)$ & $16(10)$ & $18(11)$ & \\
\hline ASA status, $n(\%)$ & & & & $0.583 \triangle$ \\
\hline 1 & $62(42)$ & $59(37)$ & $75(45)$ & \\
\hline$\|$ & $68(46)$ & $83(52)$ & $77(46)$ & \\
\hline III & $18(12)$ & $18(11)$ & 15(9) & \\
\hline Previous lower abdominal surgery, $n(\%)$ & $39(26)$ & $45(28)$ & $40(24)$ & 0.689 \\
\hline Previous PONV, $n(\%)$ & $17(11)$ & $24(15)$ & $32(19)$ & 0.167 \\
\hline \multicolumn{5}{|l|}{ Diagnosis, $n(\%)$} \\
\hline Myoma & $69(47)$ & 63(39) & $74(44)$ & 0.420 \\
\hline Adenomyosis & 29(19) & $35(22)$ & $38(23)$ & 0.784 \\
\hline Endometriosis & $18(12)$ & $22(14)$ & $14(8)$ & 0.291 \\
\hline Cervical dysplasia & $7(5)$ & $11(7)$ & 13(8) & $0.166^{*}$ \\
\hline Other benign & $25(17)$ & $29(18)$ & $28(17)$ & 0.939 \\
\hline Surgical approach (laparoscopy/laparotomy) & $28 / 120$ & $11 / 149$ & $10 / 157$ & $<0.001$ \\
\hline Preoperative hemoglobin $\left(\mathrm{g} / \mathrm{L}^{\mathrm{a}}\right)$, median (IQR) & 121(101-133) & 117(103-138) & 122(99-136) & $0.356^{\triangle}$ \\
\hline Preoperative hypokalemia incidence, $n(\%)$ & $44(30)$ & $29(18)$ & $25(15)$ & 0.003 \\
\hline Duration of operation $\left(\min ^{\mathrm{a}}\right)$, median (IQR) & $95(70-125)$ & $105(75-140)$ & $105(80-140)$ & $0.446^{\triangle}$ \\
\hline Intraoperative blood loss $\left(\mathrm{ml}^{\mathrm{a}}\right)$, median (IQR) & $50(50-100)$ & $50(50-90)$ & $50(50-100)$ & $0.609 \triangle$ \\
\hline Intraoperative net fluid input $\left(\mathrm{ml}^{\mathrm{a}}\right)$, median (IQR) & $1600(1100-2000)$ & $1600(1100-1600)$ & $1500(1000-1500)$ & $0.072^{\triangle}$ \\
\hline
\end{tabular}

Abbreviations: ASA American Society of Anesthesiologists, BMI Body mass index, COPD Chronic obstructive pulmonary disease, IQR Interquartile range, NRS Nutritional risk screening, NYHA New York Heart Association, PONV Postoperative nausea and vomiting

${ }^{a}$ Continuous variables were described as median (IQR), categorical variables as number of events $(n)$

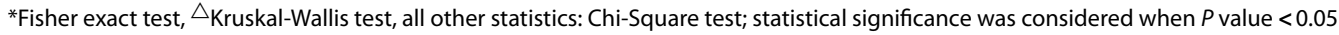


Furthermore, no clinically significant differences were observed in diagnosis, duration of operation, preoperative hemoglobin or bleeding volume. Significantly, there was a higher proportion of preoperative hypokalemia incidence in the Group I than that in groups II and III (I vs II: $30 \%$ vs $18 \%, P=0.007$; I vs III: $30 \%$ vs $15 \%, P=0.002)$. Meanwhile, the intraoperative net fluid input volume of Group III was lower than that of other groups, but the difference was not statistically significant.

\section{Postoperative complications}

Postoperative complications of the three groups were shown in Table 2. There was a significant decrease in the proportion of patients with any complication between Group I and III $(P=0.013)$. Compared with group I, the incidence of PONV and moderate-to-severe postoperative pain decreased significantly than that in the group III $(P<0.001)$. The incidence of pulmonary infection was $6.1,6.3$, and $1.8 \%(P=0.044)$. No statistically significant difference in the other complications among the three groups was observed $(P>0.05)$.

\section{QoR-15 scores and other perioperative outcomes}

No significant difference in the QoR-15 scores among the three groups for the day before operation was found (Fig. 2). Compare with Group I, the total QoR-15 scores in the groups II and III were higher in the first 3 days after surgery $(P<0.001)$. In addition, compare with Group II, the total QoR-15 scores in Group III were higher in the first 3 days after surgery $(P<0.001)$ (Fig. $2 \mathrm{~A})$.

Among the five dimensions of the QoR-15, scores of physical comfort $(P<0.05)$, physical independence
$(P<0.05)$, and pain dimension $(P<0.05)$ in the group II and III were significantly higher compared to the group I in the first 3 days after surgery; scores of emotional status $(P<0.05)$ in the group III was significantly higher than those in the group I and II in the first 2 days after surgery; scores of psychological support $(P<0.05)$ in the group III were significantly higher compared to the group I and II on POD 2(Fig. 2B).

In this study, no patients developed serious postoperative complications within 30 day after operation (such as ileus, atelectasis, myocardial infarction and bleeding complications), meanwhile no significant differences in the incidence of no-planned re-operation and postoperative hospitalization cost was observed (Table 2). One localized subcutaneous hematoma beneath the incision occurred in one patient and experienced no-planned re-operation, with no patients experiencing re-admission or died during hospitalization and within 30 days after discharge.

\section{Patient satisfaction}

Patient satisfaction on discharge day and the 30 days after discharge in each group was shown in Fig. 3. It was significantly higher in the groups II and III than that in the Group I on the discharge day (I vs II: 4(4-4) vs 5(5-6), $P<0.001$; I vs III: $4(4-4)$ vs $6(5-6), P<0.001$; II vs III:5(5-6) vs $6(5-6), P<0.001)$. In addition, patient satisfaction was also significantly higher in the groups II and III than that in the Group I on the 30 day after discharge (I vs II: $4(4-5)$ vs $5(5-6), P<0.001$; I vs III: 4 (4-5) vs 6(6-7), $P<0.001$; II vs III:5(5-6) vs $6(6-7)$, $P<0.001)$.

Table 2 Comparison of Postoperative Complications and other Perioperative Outcomes among the Groups

\begin{tabular}{|c|c|c|c|c|}
\hline Compliance & $\begin{array}{l}\mathrm{I}[0,60 \%) \\
(N=148)\end{array}$ & $\begin{array}{l}I I[60,80 \%) \\
(N=160)\end{array}$ & $\begin{array}{l}\text { III }[80,100 \%] \\
(N=167)\end{array}$ & $P$ value \\
\hline PONV, n (\%) & $39(26.3)$ & $40(25.0)$ & 26(15.6) & 0.039 \\
\hline Moderate-to-severe postoperative pain, n (\%) & 25(16.9) & 23(14.4) & $7(4.2)$ & $<0.001$ \\
\hline DVT, n (\%) & $1(0.7)$ & $1(0.6)$ & $0(0.0)$ & $0.307^{*}$ \\
\hline Surgical site infection, n (\%) & $5(3.4)$ & $5(3.1)$ & $3(1.8)$ & $0.244^{*}$ \\
\hline Pulmonary infection, n (\%) & $9(6.1)$ & $10(6.3)$ & $3(1.8)$ & $0.044^{*}$ \\
\hline Overall complications, n (\%) & $61(41.2)$ & $61(38.1)$ & $34(20.4)$ & $<0.001$ \\
\hline No-planned re-operation, $n(\%)$ & $0(0)$ & $1(1)$ & $0(0)$ & $0.373^{*}$ \\
\hline Readmission, $n(\%)$ & $0(0)$ & $0(0)$ & $0(0)$ & \\
\hline Mortality, $n(\%)$ & $0(0)$ & $0(0)$ & $0(0)$ & \\
\hline LOS after surgery $\left(d^{\mathrm{a}}\right)$, median (IQR) & $5(4-6)$ & $4(3-5)$ & $4(3-4)$ & $0.007 \triangle$ \\
\hline Postoperative hospitalization cost $\left(\right.$ Euro $\left.^{\mathrm{a}}\right)$, median (IQR) & $3856(2194)$ & $2591(2170)$ & 2453(2388) & $0.134^{\triangle}$ \\
\hline
\end{tabular}

Abbreviations: DVT Deep vein thrombosis, IQR Interquartile range, $P O D$ Postoperative day, PONV Postoperative nausea and vomiting;

${ }^{\text {a }}$ Continuous variables were described as median (IQR), categorical variables as number of events ( $n$ )

*Fisher exact test, $\triangle$ Kruskal-Wallis test and Mann-Whitney $U$ test was used, all other statistics: Chi-Square test; statistical significance was considered when $P$ value $<0.05$ 


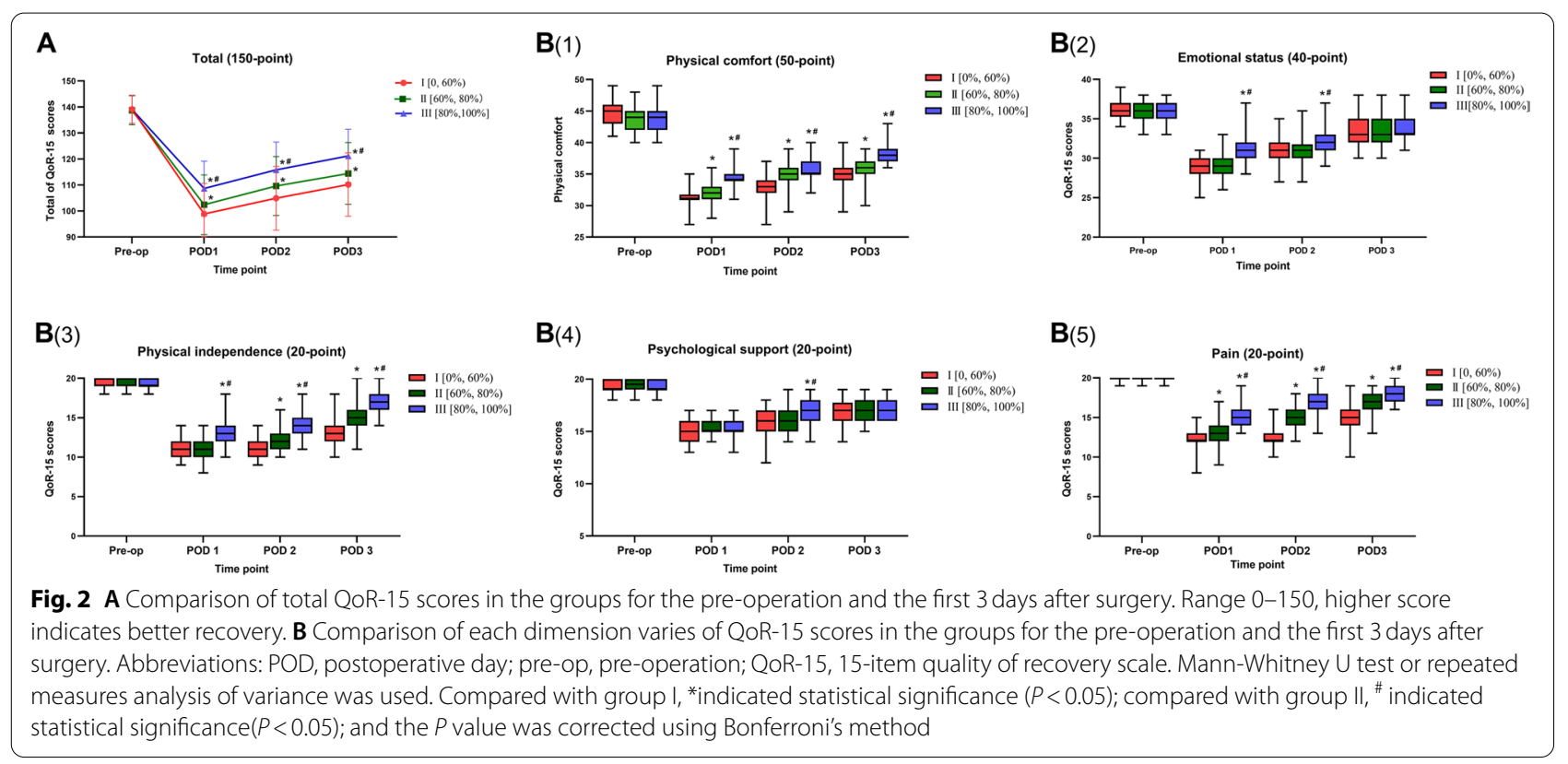

A

Patient satisfaction on discharge day

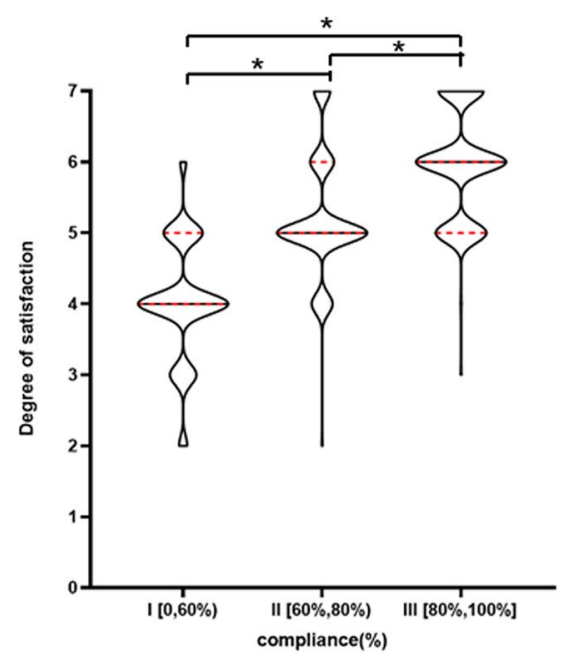

B Patient satisfaction on $30 \mathrm{~d}$ after discharge

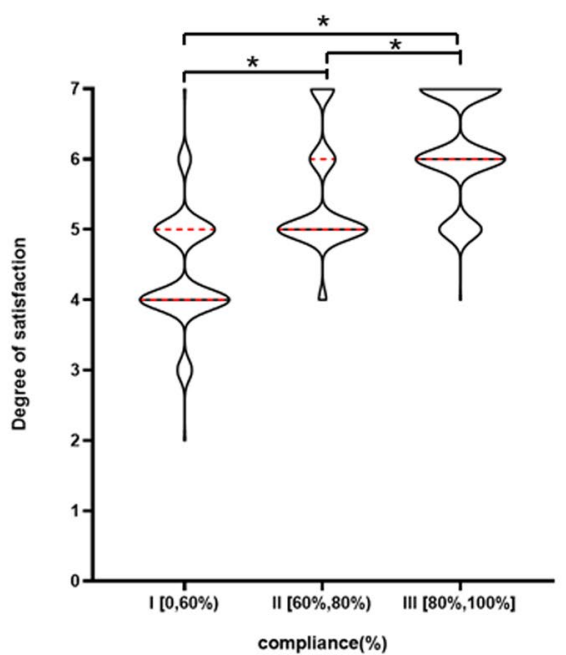

Fig. 3 Comparison of patient satisfaction in each group on discharge day $(\mathbf{A})$ and the 30 days after discharge (B). Range 0-7, higher degree indicates better patient satisfation. Mann-Whitney $U$ test was used. *Indicated statistical significance $(P<0.001)$, and the $P$ value was corrected using Bonferroni's method

\section{Association of complications and compliance with each ERAS component and the predictive nomogram development and validation}

The univariable logistic regression analysis was used to analyze the compliance with each ERAS component that might affect the probability of having any postoperative complication. (Table 3 ). The factors with $P$ values more than 0.05 were excluded from multivariate analysis, including no bowel preparation $(P=0.573)$, oral carbohydrate loading $(P=0.121)$, no abdominal drainage $(P=0.062)$, no routine nasogastric tube $(P=0.339)$, postoperative glucose control $(P=0.203)$ and peritoneal drainage $(P=0.215)$. The other factors with $P$ values less than 0.05 , were further included in the multivariable logistic regression. Finally, five factors with $P$ values less than 0.05 in multivariable regression analysis were 
Table 3 Association of having any postoperative complication within 30d and compliance with each ERAS component

\begin{tabular}{|c|c|c|c|c|c|c|}
\hline \multirow[t]{2}{*}{ ERAS component } & \multicolumn{3}{|c|}{ Univariable analysis } & \multicolumn{3}{|c|}{ Multivariable analysis } \\
\hline & OR & $95 \% \mathrm{Cl}$ & $P$-value & OR & $95 \% \mathrm{Cl}$ & $P$-value \\
\hline Education and counseling & 0.26 & $0.11-0.63$ & 0.003 & 0.25 & $0.44-1.42$ & 0.118 \\
\hline Pre-operative optimization & 0.50 & $0.33-0.75$ & 0.001 & 2.07 & $0.98-4.40$ & 0.057 \\
\hline No prolonged fasting & 2.07 & $1.09-3.94$ & 0.026 & 1.04 & $0.41-2.61$ & 0.938 \\
\hline No pre-anesthetic medication & 0.24 & $0.11-0.49$ & $<0.001$ & 0.73 & $0.17-3.11$ & 0.666 \\
\hline Standard anesthetic protocol & 0.33 & $0.21-0.52$ & $<0.001$ & 0.71 & $0.25-2.06$ & 0.530 \\
\hline Minimally invasive surgery & 0.18 & $0.09-0.33$ & $<0.001$ & 0.13 & $0.05-0.34$ & $<0.001$ \\
\hline Goal-directed fluid therapy & 0.49 & $0.32-0.75$ & 0.001 & 0.60 & $0.32-1.11$ & 0.101 \\
\hline Maintenance of normothermia & 0.19 & $0.04-0.98$ & 0.048 & 0.67 & $0.03-9.97$ & 0.812 \\
\hline PONV prophylaxis & 0.06 & $0.03-0.12$ & $<0.001$ & 0.05 & $0.02-0.12$ & $<0.001$ \\
\hline Multimodal prevention of DVT & 0.52 & $0.35-0.77$ & 0.001 & 1.08 & $0.55-2.15$ & 0.824 \\
\hline Avoid salt-water overload & 1.89 & $1.11-3.20$ & 0.019 & 1.36 & $0.61-3.01$ & 0.454 \\
\hline Multimodal analgesia & 0.38 & $0.25-0.57$ & $<0.001$ & 0.97 & $0.37-2.54$ & 0.963 \\
\hline Early mobilization & 0.28 & $0.19-0.43$ & $<0.001$ & 0.50 & $0.27-0.94$ & 0.031 \\
\hline Early oral intake & 0.21 & $0.13-0.32$ & $<0.001$ & 0.37 & $0.17-0.81$ & 0.012 \\
\hline Urinary drainage & 0.08 & $0.05-0.13$ & $<0.001$ & 0.06 & $0.03-0.12$ & $<0.001$ \\
\hline
\end{tabular}

Abbreviations: Cl Confidence interval, DVT Deep vein thrombosis, ERAS Enhanced recovery after surgery, OR Odds ratio, PONV Postoperative nausea and vomiting

recruited to construct the prediction model, including minimally invasive surgery $(p<0.001)$, PONV prophylaxis with over 2 antiemetic agents $(p<0.001)$, early mobilization $(p=0.031)$, early oral intake $(p=0.012)$, and early removal of urinary drainage $(p<0.001)$.

The nomogram prediction model was established with 5 independent factors to predict the risk of postoperative complications. As shown in Fig. 4, each factor corresponds to a specific point by drawing a line straight upward to the Points axis. The probability of postoperative complication is the point by drawing a line straight down to the bottom axis from the sum of the points on the Total Points axis. For example, according to the model, a woman who underwent minimally invasive surgery ( 0 point) treated with early oral intake ( 0 point $)$ and early urinary drainage ( 0 point), but not with PONV prophylaxis (100 point) and early mobilization (20 point) with about $67 \%$ incidence of postoperative complications. The validation of the the nomogram prediction model was based on calibration and discrimination. The calibration curve demonstrated a good calibration because the actual line was not significantly deviated from the ideal line. The result of ROC curve showed an excellent discrimination with a high AUC value (0.906) and sensitivity (0.948).

\section{Discussion}

Implementation of the ERAS pathway remains an ongoing challenge in clinical practice which requires the engagement of nursing team, surgical team, and patients. Individual ERAS components are implemented at different stages during the patient's hospitalization, which may adversely influence compliance. Furthermore, implementation of ERAS pathway is associated with a shift in clinical routines, from old practices to new pathway. This study is the first study to our knowledge determining the effect of the compliance of patients to an ERAS protocol after benign hysterectomy on outcomes with the QoR-15 scales and patient satisfaction. Our current study indicated that an overall compliance rate of 80.6\% for ERAS in patients undergoing hysterectomy. Similarly, a recent observational study reported a mean compliance rate of $77 \%$ after the ERAS program was implemented in gynecologic oncology [29]. Consistent with other studies, improved adherence to the ERAS protocols improved clinical rehabilitation $[4,5,30]$, we found patients with compliance over $80 \%$ had a significant reduction in postoperative complications and length of stay after surgery. Increased compliance was also associated with higher patient satisfaction and QoR-15 scores. The difference in the incidence of postoperative complications within 30 days between the lowest and highest compliance groups was $20.8 \%$.

Most previous studies evaluating postoperative recovery have focused on physiological parameters such as LOS and morbidity [22, 31-34]. As the recovery process is complex and encompasses the multiple dimensions of physical [35], emotional and social health, patients' reported outcome are essential to evaluate the quality of recovery, measuring any aspect of a patient's health status with information derived directly from the patient. Patient's health status was measured 


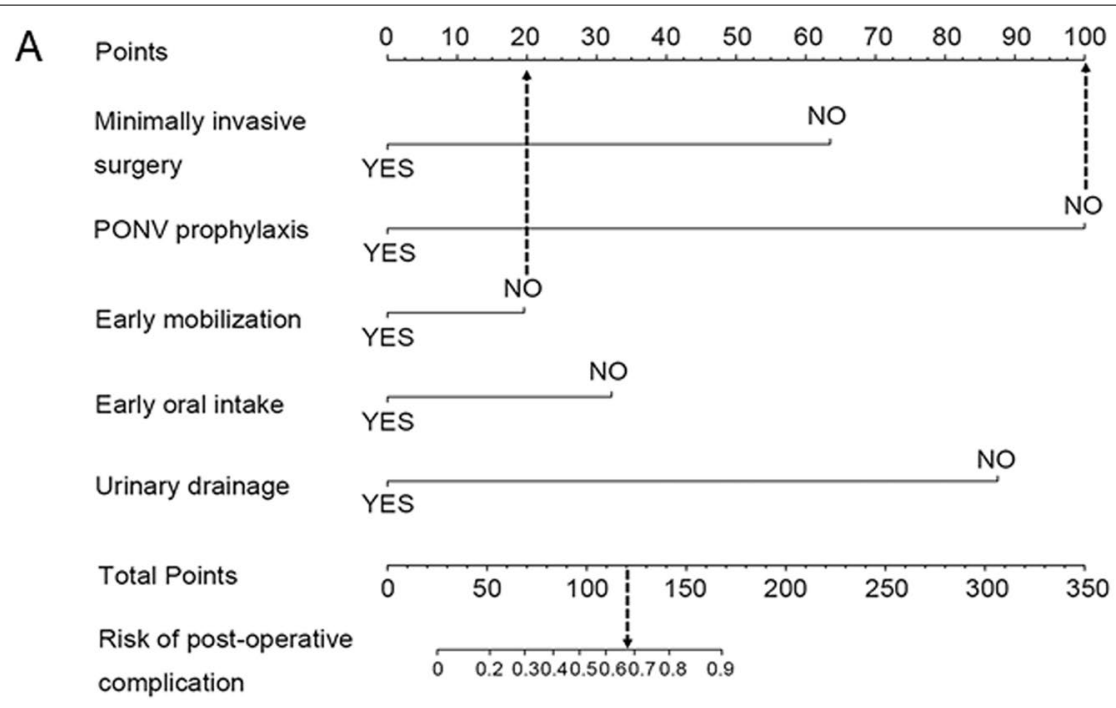

B

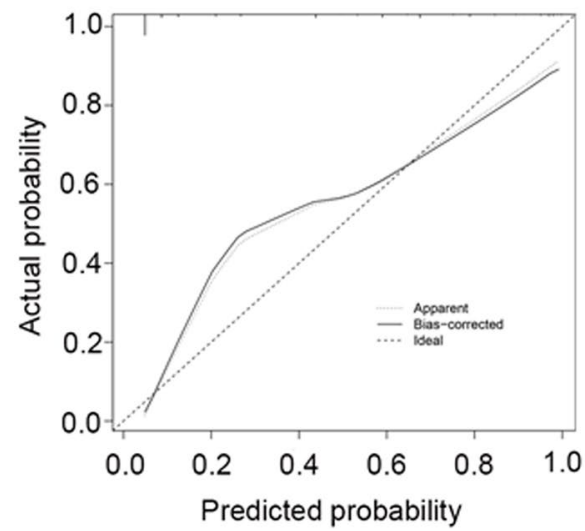

C

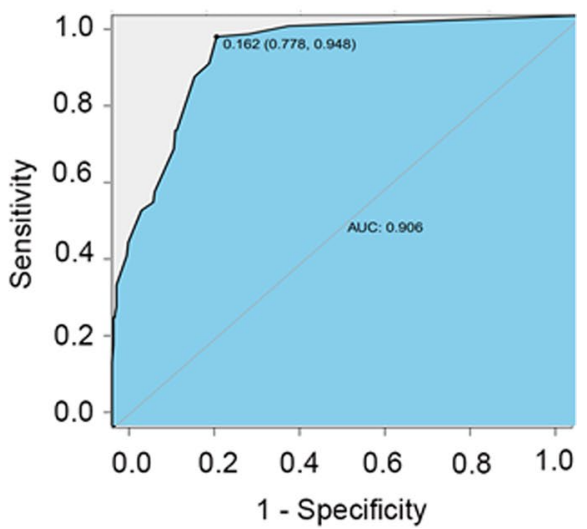

Fig. 4 Nomogram to predict risk of postoperative complication. A Each factor corresponds to a specific point by drawing a line straight upward to the Points axis. The probability of postoperative complication is the point by drawing a line straight down to the bottom axis from the sum of the points on the Total Points axis. Calibration curve and ROC curve of the nomogram prediction model. B Calibration curve. The dashed line represents the ideal fit; the solid line represents the actual fit $(\mathbf{C})$ ROC curve. (AUC $=0.906)$

with the QoR-15 scale and patient satisfaction. Meanwhile Chinese version QoR-15 has good reliability, validity, clinical acceptability and feasibility $[23,24,36]$.

We found the QoR-15 scores and patient satisfaction were significantly higher in the higher compliance group, which can be explained by the reduction pain and improvement mood during the early postoperative period. In the ERAS protocols, preoperative information education, no prolonged fasting, no bowel preparation and oral carbohydrate loading can relieve the patient discomfort such as anxiety, hungry and thirsty. In addition to the above advantages, it also increases patient comfort and physical independence by reducing the incidence of postoperative nausea and vomiting, relieving pain and accelerating the recovery. The increase in patient satisfaction after multimodal analgesic approaches may be a significant benefit, along with a reduction in postoperative complications. In order to avoid the interference of other factors on the results, we really scored the patients, and we chose to conduct the satisfaction survey of the patients on the day of discharge and 30 days after discharge.

Suggestions for improving compliance include the use of a dedicated wards, specific personnel, effective education and training, and regular inspections [37]. The lack of repeated education on ERAS protocols has a significant influence on compliance, as well as analyzing the obstacles and catalysts to implementation and compliance. Every participant needs to know the implementation of ERAS protocols can be challenging, but ultimately rewarding [38]. 
No serious complications were observed in this study. However, due to our relatively small trial size, which limited our ability to judge the frequency of rare but potentially serious events. PONV is still the main problem affecting the early postoperative feeding and activity of patients. General anesthesia combined with TAP block and multimodal postoperative analgesia pathway could reduce the use of opioids, meanwhile reduce the occurrence of PONV [39]. At present, the relationship between major complications and compliance with ERAS remains the focus of research [40].

We also noted that the incidence of preoperative hypokalemia was higher in patients with $<60 \%$ compliance, it may be attributed to the no prolonged fasting, as well as insulin resistance associated with oral carbohydrate intake, which deserved further research. Several limitations of the current study need to be addressed. First, a randomized controlled trial should be the preferred option, yet now the existing evidence on ERAS protocols; we considered it unethical to conduct a randomized controlled trial. However, the relatively large sample size, the fixed ERAS MDT and the prospective data collection by a dedicated nurse are strengths of this study. Although most data were entered prospectively, analysis was done retrospectively and results should be interpreted as such. Further researches into the ERAS pathway are needed, including using validated international classification systems such as Clavien-Dindo, and evaluation of additional patient-related outcomes such as patient experience of the process and longer-term consequences. In addition, the predictive nomogram model without a subsequent validation study, it cannot be extrapolated. And the validation and optimization of current model needed to be performed in future study.

\section{Conclusion}

In summary, better compliance to the ERAS protocols modified for gynecological surgery is crucial to postoperative quality of recovery after benign hysterectomy, decrease the overall complication rate and improve patient experience without a significant increase in readmission and mortality. In this study in particular, minimally invasive surgery, PONV prophylaxis, early mobilization, early oral intake, and early removal of urinary drainage were associated with a lower complication rate after benign hysterectomy.

\footnotetext{
Abbreviations

ASA: American Society of Anesthesiologists; BMI: Body mass index; Cl: Confidence interval; COPD: Chronic obstructive pulmonary disease; DVT: Deep vein thrombosis; ERAS: Enhanced recovery after surgery; NRS: Nutritional risk screening; MDT: Multi-disciplinary team; NYHA: New York Heart Association;
}

POD: Postoperative day; PONV: Postoperative nausea and vomiting; QoR-15: 15-item quality of recovery scale.

\section{Supplementary Information}

The online version contains supplementary material available at https://doi. org/10.1186/s12871-021-01509-0.

Additional file 1. ERAS protocol used in our department.

Additional file 2. QoR-15 questionnaire used in the study.

Additional file 3. Definition of postoperative complications in the study.

\section{Acknowledgments}

The authors thank all the staff in this study for their dedication, support and hard work.

\section{Authors' contributions}

SM and YWS designed the current study. JYJ verified the analytical methods. YWS, PPQ, YG, YZ, GMW, FL carried out the project and collected all of the data. $F L, J Y, P P Q$ analyzed the data. YWS wrote the manuscript with support from SM, FL and JYJ. All authors edited, read, and approved the final version of the manuscript.

\section{Funding}

This work has received funding from the National Key Clinical Specialty Construction Project (2011-170) and Chongqing Social Science Planning Project (2021NDQN55); and also received support from the Xinchen Cup Cultivation Project of perioperative Anesthesia and ERAS in Chongqing (2018-MZ-2).

\section{Availability of data and materials}

The datasets used and analyzed in this study is available from the corresponding author on reasonable request.

\section{Declarations}

Ethics approval and consent to participate

This study was approved and conducted by the local Ethics Committee of the First Affiliated Hospital of Chongqing Medical University (No.2019-020), and registered with ClinicalTrials.gov (ChiCTR1800019178, 30/10/2018). No personally identifying information is included in this manuscript.

Consent for publication

Not applicable.

Competing interests

All the authors declared no potential conflicts of interest with respect to the research, authorship, and/or publication of this article.

\section{Author details}

${ }^{1}$ Department of Anesthesiology, The First Affiliated Hospital of Chongqing Medical University, No.1 Youyi Road, Yuzhong District, Chongqing 400016, People's Republic of China. ${ }^{2}$ Department of Gynecology, the First Affiliated Hospital of Chongqing Medical University, Chongqing, China.

Received: 1 September 2021 Accepted: 9 November 2021

Published online: 22 November 2021

\section{References}

1. Fearon $\mathrm{KC}$, Ljungqvist $\mathrm{O}$, Von Meyenfeldt M, Revhaug A, Dejong $\mathrm{CH}$, Lassen $\mathrm{K}$, et al. Enhanced recovery after surgery: a consensus review of clinical care for patients undergoing colonic resection. Clin Nutr. 2005:24:466-77.

2. Wijk L, Udumyan R, Pache B, Altman AD, Williams LL, Elias KM, et al. International validation of enhanced recovery after surgery society guidelines 
on enhanced recovery for gynecologic surgery. Am J Obstet Gynecol. 2019;221:237.e1-237.e11.

3. Kehlet H. Multimodal approach to control postoperative pathophysioogy and rehabilitation. Br J Anaesth. 1997;78:606-17.

4. Gustafsson UO, Hausel J, Thorell A, Ljungqvist O, Soop M, Nygren J. Enhanced recovery after surgery study group: adherence to the enhanced recovery after surgery protocol and outcomes after colorectal cancer surgery. Arch Surg. 2011;146:571-7.

5. Cakir H, van Stijn MF, Lopes Cardozo AM, Langenhorst BL, Schreurs WH, van der Ploeg TJ, et al. Adherence to enhanced recovery after surgery and length of stay after colonic resection. Color Dis. 2013;15:1019-25.

6. Wang W, Wang Y, Wu H, Lei L, Xu S, Shen $X$, et al. Postoperative cognitive dysfunction: current developments in mechanism and prevention. Med Sci Monit. 2014;20:1908-12.

7. Pędziwiatr M, Mavrikis J, Witowski J, Adamos A, Major P, Nowakowski M, et al. Current status of enhanced recovery after surgery (ERAS) protocol in gastrointestinal surgery. Med Oncol. 2018;35:95.

8. Pisarska M, Pędziwiatr M, Major P, Kisielewski M, Migaczewski M, Rubinkiewicz M, et al. Laparoscopic Gastrectomy with enhanced recovery after surgery protocol: single-center experience. Med Sci Monit. 2017;23:1421-7.

9. Proudfoot S, Bennett B, Duff S, Palmer J. Implementation and effects of enhanced recovery after surgery for hip and knee replacements and fractured neck of femur in New Zealand orthopaedic services. N Z Med J. 2017;130:77-90.

10. Hammer A, Rositch AF, Kahlert J, Gravitt PE, Blaakaer J, Søgaard M. Global epidemiology of hysterectomy: possible impact on gynecological cancer rates. Am J Obstet Gynecol. 2015;213:23-9.

11. Bowyer A, Jakobsson J, Ljungqvist O, Royse C. A review of the scope and measurement of postoperative quality of recovery. Anaesthesia. 2014;69:1266-78.

12. Kroon UB, Rådström M, Hjelthe C, Dahlin C, Kroon L. Fast-track hysterectomy: a randomised, controlled study. Eur J Obstet Gynecol Reprod Biol. 2010;151:203-7.

13. Carter J. Fast-track surgery in gynaecology and gynaecologic oncology: a review of a rolling clinical audit. ISRN Surg. 2012;2012:368014.

14. Bernard L, McGinnis JM, Su J, Alyafi M, Palmer D, Potts L, et al. Thirty-day outcomes after gynecologic oncology surgery: a single-center experience of enhanced recovery after surgery pathways. Acta Obstet Gynecol Scand. 2021;100:353-61.

15. Bisch SP, Jago CA, Kalogera E, Ganshorn H, Meyer LA, Ramirez PT, et al. Outcomes of enhanced recovery after surgery (ERAS) in gynecologic oncology - a systematic review and meta-analysis. Gynecol Oncol. 2021:161:46-55

16. Marret $H$, Fritel X, Ouldamer L, Bendifallah S, Brun JL, De Jesus I, et al. Therapeutic management of uterine fibroid tumors: updated French guidelines. Eur J Obstet Gynecol Reprod Biol. 2012;165:156-64.

17. Chrysostomou A, Djokovic D, Edridge W, van Herendael BJ. Evidencebased guidelines for vaginal hysterectomy of the International Society for Gynecologic Endoscopy (ISGE). Eur J Obstet Gynecol Reprod Biol. 2018:231:262-7.

18. Nelson G, Altman AD, Nick A, Meyer LA, Ramirez PT, Achtari C, et al. Guidelines for pre- and intra-operative care in gynecologic/oncology surgery: enhanced recovery after surgery $\left(\right.$ ERAS $\left.^{\circledR}\right)$ society recommendations--part I. Gynecol Oncol. 2016;140:313-22.

19. Nelson G, Altman AD, Nick A, Meyer LA, Ramirez PT, Achtari C, et al. Guidelines for postoperative care in gynecologic/oncology surgery: enhanced recovery after surgery $\left(\right.$ ERAS $\left.^{\circledR}\right)$ society recommendations--part II. Gynecol Oncol. 2016;140:323-32.

20. Nelson G, Bakkum-Gamez J, Kalogera E, Glaser G, Altman A, Meyer $L A$, et al. Guidelines for perioperative care in gynecologic/oncology: enhanced recovery after surgery (ERAS) society recommendations-2019 update. Int J Gynecol Cancer. 2019;29:651-68.

21. Wong-Lun-Hing EM, van Dam RM, Heijnen LA, Busch OR, Terkivatan T, van Hillegersberg $R$, et al. Is current perioperative practice in hepatic surgery based on enhanced recovery after surgery (ERAS) principles. World J Surg. 2014:38:1127-40

22. Iniesta MD, Lasala J, Mena G, Rodriguez-Restrepo A, Salvo G, Pitcher B, et al. Impact of compliance with an enhanced recovery after surgery pathway on patient outcomes in open gynecologic surgery. Int J Gynecol Cancer. 2019;29:1417-24.

23. Stark PA, Myles PS, Burke JA. Development and psychometric evaluation of a postoperative quality of recovery score: the QoR-15. Anesthesiology. 2013:118:1332-40.

24. Chazapis M, Walker EM, Rooms MA, Kamming D, Moonesinghe SR. Measuring quality of recovery-15 after day case surgery. $\mathrm{Br} J$ Anaesth. 2016;116:241-8.

25. Miller TE, Mythen M. Successful recovery after major surgery: moving beyond length of stay. Perioper Med (Lond). 2014;3:4.

26. Wang S, Gao PF, Guo X, Xu Q, Zhang YF, Wang GQ, et al. Effect of lowconcentration carbohydrate on patient-centered quality of recovery in patients undergoing thyroidectomy: a prospective randomized trial. BMC Anesthesiol. 2021;21:103.

27. Park JY, Hong JH, Kim DH, Yu J, Hwang JH, Kim YK. Magnesium and bladder discomfort after transurethral resection of bladder tumor. Anesthesiology. 2020;133:64-77.

28. Jammer I, Wickboldt N, Sander M, Smith A, Schultz MJ, Pelosi P, et al. Standards for definitions and use of outcome measures for clinical effectiveness research in perioperative medicine: European perioperative clinical outcome (EPCO) definitions: a statement from the ESA-ESICM joint taskforce on perioperative outcome measures. Eur J Anaesthesiol. 2015;32:88-105.

29. Bisch SP, Wells T, Gramlich L, Faris P, Wang X, Tran DT, et al. System-wide implementation and audit leads to improved value and patient outcomes. Gynecol Oncol. 2018;151:117-23.

30. Feroci F, Lenzi E, Baraghini M, Garzi A, Vannucchi A, Cantafio S, et al. Fasttrack colorectal surgery: protocol adherence influences postoperative outcomes. Int J Color Dis. 2013;28:103-9.

31. Gentry ZL, Boitano T, Smith HJ, Eads DK, Russell JF, Straughn JM Jr. The financial impact of an enhanced recovery after surgery (ERAS) protocol in an academic gynecologic oncology practice. Gynecol Oncol. 2020;156:284-7.

32. Dickson EL, Stockwell E, Geller MA, Vogel RI, Mullany SA, Ghebre R, et al. Enhanced recovery program and length of stay after laparotomy on a gynecologic oncology service: a randomized controlled trial. Obstet Gynecol. 2017;129:355-62.

33. Marx C, Rasmussen T, Jakobsen DH, Ottosen C, Lundvall L, Ottesen B, et al. The effect of accelerated rehabilitation on recovery after surgery for ovarian malignancy. Acta Obstet Gynecol Scand. 2006;85:488-92.

34. Wijk L, Franzen K, Ljungqvist O, Nilsson K. Implementing a structured enhanced recovery after surgery (ERAS) protocol reduces length of stay after abdominal hysterectomy. Acta Obstet Gynecol Scand. 2014;93:749-56.

35. Lee L, Tran T, Mayo NE, Carli F, Feldman LS. What does it really mean to "recover" from an operation. Surgery. 2014;155:211-6.

36. Bu XS, Zhang J, Zuo YX. Validation of the Chinese version of the quality of recovery-15 score and its comparison with the post-operative quality recovery scale. Patient. 2016;9:251-9.

37. Ahmed J, Khan S, Lim M, Chandrasekaran TV, MacFie J. Enhanced recovery after surgery protocols - compliance and variations in practice during routine colorectal surgery. Color Dis. 2012;14:1045-51.

38. Ellis DB, Agarwala A, Cavallo E, Linov P, Hidrue MK, Del Carmen MG, et al. Implementing ERAS: how we achieved success within an anesthesia department. BMC Anesthesiol. 2021;21:36.

39. Osborn S, Cyna AM, Middleton P, Griffiths JD. Perioperative transversus abdominis plane (TAP) blocks for analgesia after abdominal surgery. Cochrane Database Syst Rev. 2020;4:CD007705.

40. Ramirez-Rodriguez JM, Martinez-Ubieto J, Muñoz-Rodes JL, RodriguezFraile JR, Garcia-Erce JA, Blanco-Gonzalez J, et al. Surgical treatment for colorectal cancer: analysis of the influence of an enhanced recovery programme on long-term oncological outcomes-a study protocol for a prospective, multicentre, observational cohort study. BMJ Open. 2020:10:e040316

\section{Publisher's Note}

Springer Nature remains neutral with regard to jurisdictional claims in published maps and institutional affiliations. 